\title{
Conditions for observation of Rabi oscillations in an exciton-polariton system
}

\author{
A.S. Shumovsky ${ }^{1}$, Ö.E. Müstecaplioğlu \\ Department of Physics, Bilkent University, Bilkent, 06533 Ankara, Turkey
}

Received 13 September 1995; accepted for publication 27 September 1995

Communicated by V.M. Agranovich

\begin{abstract}
The pure quantum model describing Rabi oscillations of exciton-polaritons (EP) in a micro-cavity with finite quality is considered. It is shown that the oscillations can be observed if the cavity damping rate does not exceed some critical value depending on the EP coupling constant and the detuning of the cavity mode. An explicit expression for the renormalized Rabi frequency is found. Comparison of the results with experimental data shows a good agreement.
\end{abstract}

PACS: 42.50. - p; 42.55.Px; 42.60.Da

Keywords: Rabi oscillations; Excitons; Polaritons; Microcavities

\section{Introduction}

The study of vacuum-field induced Rabi oscillations in various physical systems is now an active field of research due to its importance in the investigation of the spontaneous emission rate in a cavity and plays a crucial part in the development of new light emitting devices with special properties, as for instance narrow linewidth, low-threshold pump-rate, and low level of noise (see, e.g., Ref. [1]). In recent years, it has become possible to observe the Rabi oscillations not only for trapped atoms [2-4] but also in a semiconductor micro-cavity [5].

The simplest system showing Rabi oscillations as the process in which the emitter and radiation field pass energy back and forth periodically, is the Jaynes-Cummings model for a two-level atom in an ideal cavity (see, e.g., Ref. [6]). In the case of solids an important example of a two-level system is provided by two branches of a polariton formed by coupled modes of the radiation field and collective excitations of solids [7]. Unlike the Jaynes-Cummings model, the occupation number of any level of that two-level system can take an arbitrary integer value due to the bosonic nature of the polariton. An important example is provided by an exciton-polari-

\footnotetext{
'Also at: Bogolubov Laboratory for Theoretical Physics, JINR, Dubna, Russian Federation.
} 
ton (EP) [8]. The possibility of direct observation of the Rabi oscillations in an EP system has been experimentally confirmed in recent publications [9-12].

The system examined in Refs. [9-12] represents a multiple-quantum-well structure placed in the center of a Fabry-Perot micro-cavity. The parameters of the micro-cavity were chosen to obey the resonance condition for the cavity and EP modes. Strong beats with the period of Rabi oscillations were observed for the pumping field tuned in resonance with the cavity mode while the detuning from resonance led to a strong reduction of beats [9].

Because of the simplicity of the EP system described by a bilinear boson Hamiltonian, it is not hard to construct the theory of the process under consideration $[8,13]$. Here we turn our attention to the consideration of two important problems which in our opinion have not been examined in sufficient detail. Precisely, in Section 2, we consider the pumping process and determine the state of the EP system formed by an external light pulse. Then, in Section 3, we examine the effects of damping due to the low-quality cavity. In this way we define the optimum conditions of observation of the Rabi oscillations in the EP system.

\section{Preparation of the exciton-polariton system}

As the EP system in a quantum-well micro-cavity is usually examined at low temperature [9-13], it is natural to consider this system initially in the vacuum state of the exciton-polariton field. The excitons in the micro-cavity are created by an external light pulse. Since the period of the Rabi oscillation is of the order of a few hundred fs, the pulse duration should be considerably shorter. In a typical experiment, pulses with 80 fs duration are used [9]. As long as the pump-system interaction is stronger than the damping rate we can safely ignore the damping effects during the action of the pumping pulse on the system.

In order to take into account the principal peculiarities of the EP system and not to go into detail let us restrict our consideration to the model Hamiltonian of the following form,

$$
H=H_{\mathrm{EP}}+H_{\mathrm{P}}(t), \quad H_{\mathrm{EP}}=\omega a^{\dagger} a+\epsilon b^{\dagger} b+g\left(a^{\dagger} b+b^{\dagger} a\right), \quad H_{\mathrm{P}}(t)=\Omega(t)\left(\mathrm{e}^{\mathrm{i} \omega_{01} t} b+\mathrm{e}^{-i \omega_{01} t} b^{\dagger}\right) .
$$

Here for simplicity we chose the EP Hamiltonian in the rotating wave approximation. The operators $a, a^{\dagger}$ and $b, b^{\dagger}$ describe the cavity photons and excitons, respectively, $\omega$ is the frequency of the cavity mode, $\epsilon$ is the exciton frequency, and $g$ is the exiton-photon coupling constant. The second term in Eq. (1) describes the time-dependent pumping by a classical field with the frequency $\omega_{0}$ and Rabi frequency $\Omega(t)$.

Using the standard procedure of the Bogolubov canonical transformation,

$$
c_{\lambda}=u_{\lambda} a+v_{\lambda} b, \quad u_{\lambda}=\frac{g}{\sqrt{\left(E_{\lambda}-\omega\right)^{2}+g^{2}}}, \quad E_{ \pm}=\omega-\frac{1}{2} \Delta \pm \frac{1}{2} \Omega_{\mathrm{R}},
$$

one can transform the Hamiltonian (1) into the polariton representation,

$$
H_{\mathrm{EP}}^{c}=\sum_{\lambda} E_{\lambda} c_{\lambda}^{\dagger} c_{\lambda}, \quad H_{\mathrm{P}}^{c}(t)=\Omega(t) \sum_{\lambda}\left[\kappa_{\lambda}(t) c_{\lambda}+\text { h.c. }\right], \quad \kappa_{\lambda}(t)=\lambda u_{-\lambda} \mathrm{e}^{\mathrm{i} \omega_{0} t^{t}}
$$

Here the index $\lambda \equiv \pm$ sets the EP branches with energy $E_{\lambda}$, the Rabi frequency of the EP system $\Omega_{\mathrm{R}}$ is defined as follows,

$$
\Omega_{\mathrm{R}} \equiv \sqrt{4 g^{2}+\Delta^{2}},
$$

$\Delta \equiv \omega-\epsilon$ is the detuning of the cavity mode, and the parameters of the transformation (2) obey the condition $u_{\lambda}^{2}+v_{\lambda}^{2}=1$. 
Consider for simplicity a rectangular pulse with amplitude $\mathscr{E}_{0}$ and duration $t_{\mathrm{D}}$. Then

$$
\begin{aligned}
\Omega(t) & =g \mathscr{E}_{0}, & & \text { if } 0 \leqslant t \leqslant t_{\mathrm{D}}, \\
& =0, & & \text { otherwise. }
\end{aligned}
$$

The time-dependent perturbation of the initial vacuum state of the EP system due to the pumping is described by the following expression,

$$
|\psi(t)\rangle=\prod_{\lambda} D_{\lambda}\left(\alpha_{\lambda}(t)\right)|0\rangle_{\lambda}
$$

where $|0\rangle_{\lambda}$ denotes the vacuum state of the corresponding EP branch and $D_{\lambda}$ formally coincides with the Glauber displacement operator with

$$
\alpha_{\lambda}(t)=\lambda \frac{u_{-\lambda} g \mathscr{E}_{0}}{E_{\lambda}-\omega_{0}}\left(\mathrm{e}^{\mathrm{i}\left(E_{\lambda}-\omega_{0}\right) r}-1\right) .
$$

In other words, the Hamiltonian (3) describes the evolution of a two-mode displaced harmonic oscillator. Therefore, taking into account that the pulse duration $t_{\mathrm{D}}$ is sufficiently small with respect to the period of Rabi oscillations [9], we can suppose that by termination of the pulse the EP system is prepared in a two-mode coherent state described by the following wave function,

$$
\left|\psi\left(t_{\mathrm{D}}\right)\right\rangle=\prod_{\lambda} D_{\lambda}\left(\alpha_{\lambda}\left(T_{\mathrm{D}}\right)\right)|0\rangle_{\lambda} .
$$

Let us return now to the transformation (2). It can be represented as follows,

$$
\begin{aligned}
& c_{+}=Z^{\dagger}\left(\zeta_{+}\right) a Z\left(\zeta_{+}\right), \\
& c_{-}=Z^{\dagger}\left(\zeta_{-}\right) b Z\left(\zeta_{-}\right)
\end{aligned}
$$

where $Z\left(\zeta_{\lambda}\right) \equiv \exp \left[-\mathrm{i} \zeta_{\lambda}\left(a^{+} b-b^{+} a\right)\right]$ and $\zeta_{+}=\mathrm{i} \arctan \left(v_{+} / u_{+}\right), \zeta_{-}=\zeta_{+}-\mathrm{i} \pi$.

Representation (5) permits us to establish a relation between the EP states and the states of the photonexciton system. First of all, it follows from Eq. (5) that the EP vacuum state $|0\rangle_{\mathrm{EP}}$ defined by the stability condition

$$
\forall \lambda \quad c_{\lambda}|0\rangle_{\mathrm{EP}}=0
$$

exactly coincides with the photon-exciton vacuum state $|0\rangle_{a b} \equiv|0\rangle_{a} \otimes|0\rangle_{b}$. Consider now the EP state with given numbers of polaritons in two branches $|n, m\rangle_{\mathrm{EP}}$. Employing relation (5) then gives

$$
\begin{aligned}
|n, m\rangle_{\mathrm{EP}}= & \frac{\left(c_{+}^{\dagger}\right)^{n}\left(c_{-}^{\dagger}\right)^{m}}{\sqrt{n ! m !}} Z\left(\zeta_{+}\right) Z\left(\zeta_{-}\right)|0,0\rangle_{\mathrm{ab}} \\
= & \sum_{k=0}^{n} \sum_{j=0}^{m}(-1)^{m-j}\left(\begin{array}{c}
n \\
k
\end{array}\right)\left(\begin{array}{c}
m \\
j
\end{array}\right) v_{+}^{n+j-k} u_{+}^{m+k-j} \sqrt{\frac{(k+j) !(n+m-k-j) !}{n ! m !}} \\
& \times|k+j, n+m-k-j\rangle_{\mathrm{ab}} .
\end{aligned}
$$

Thus, the EP number state is a linear combination of a finite number of photon-exciton number states with weights taking into account the correlations between photons and excitons in the EP system. However, the coherent state of EP system corresponds to the coherent states of both photons and excitons with parameters $\alpha_{\mathrm{a}}=\alpha_{+} u_{+}+\alpha_{-} u_{-}$and $\alpha_{\mathrm{b}}=\alpha_{+} v_{+}+\alpha_{-} v_{-}$, respectively, where $\alpha_{ \pm}$is the parameter of the coherent state of the corresponding EP branch. Therefore, the light state immediately after the pulse finishes, which should be considered as the initial state due to our supposition, is exactly the coherent state described by the parameter $\alpha_{\mathrm{a}}$. 
The time-dependent number of photons is described by the relation

$$
\left\langle a^{\dagger} a\right\rangle_{t}=u_{+}^{2}\left\langle c_{+}^{\dagger} c_{+}\right\rangle_{t}+u_{-}^{2}\left\langle c_{-}^{\dagger} c_{-}\right\rangle_{t}+2 u_{+} u_{-} \operatorname{Re}\left(\left\langle c_{+}^{\dagger} c_{-}\right\rangle_{r}\right) .
$$

Therefore, one can easily see that under the condition

$$
\delta-\frac{1}{2} \Delta \pm \frac{1}{2} \Omega_{\mathrm{R}}=2 k \pi / t_{\mathrm{D}},
$$

Rabi oscillations do not occur in the system under consideration and the mean photon number is independent of time,

$$
\begin{aligned}
\left\langle a^{\dagger} a\right\rangle_{t} & =u_{+}^{2}\left|\alpha_{+}\left(t_{\mathrm{D}}\right)\right|^{2} \quad \text { if } \delta-\frac{1}{2} \Delta+\frac{1}{2} \Omega_{\mathrm{R}}=2 k \pi / t_{\mathrm{D}}, \\
& =u_{-}^{2}\left|\alpha_{-}\left(t_{\mathrm{D}}\right)\right|^{2} \quad \text { if } \delta-\frac{1}{2} \Delta-\frac{1}{2} \Omega_{\mathrm{R}}=2 k \pi / t_{\mathrm{D}} .
\end{aligned}
$$

The general expression for the mean photon number has the form

$$
\begin{aligned}
\left\langle a^{\dagger} a\right\rangle_{t}= & \frac{2 u_{-}^{2} g^{2} \mathscr{E}^{2}}{\left(\delta-\frac{1}{2} \Delta+\frac{1}{2} \Omega_{\mathrm{R}}\right)^{2}}\left[1-\cos \left(\delta-\frac{1}{2} \Delta+\frac{1}{2} \Omega_{\mathrm{R}}\right) t_{\mathrm{D}}\right] \\
& +\frac{2 u_{+}^{2} g^{2} \mathscr{E}^{2}}{\left(\delta-\frac{1}{2} \Delta-\frac{1}{2} \Omega_{\mathrm{R}}\right)^{2}}\left[1-\cos \left(\delta-\frac{1}{2} \Delta-\frac{1}{2} \Omega_{\mathrm{R}}\right) t_{\mathrm{D}}\right] \\
& -\frac{8 u_{+}^{2} u_{-}^{2} g^{2} \mathscr{E}^{2}}{g^{2}-\delta(\delta-\Delta)} \sin \left[\left(\delta+\frac{1}{2} \Delta+\frac{1}{2} \Omega_{\mathrm{R}}\right) t_{\mathrm{D}}\right] \sin \left[\left(\delta-\frac{1}{2} \Delta-\frac{1}{2} \Omega_{\mathrm{R}}\right) t_{\mathrm{D}}\right] \cos \left[\Omega_{\mathrm{R}}\left(t-\frac{1}{2} t_{\mathrm{D}}\right)\right]
\end{aligned}
$$

One can see that unlike the Jaynes-Cummings model expression (11) does not describe the collapse-revival phenomena in spite of the initial coherent state of light. Calculating the coherence rate, it is not hard to see that for any time $t$ the coherence of radiation is conserved.

The above obtained results are valid for an ideal system without losses. We now turn to the case of a damped EP system in a cavity.

\section{Radiation damping}

In order to take into account the radiation damping, we use the Heisenberg-Langevin approach [14]. Then, for the time-dependent EP operators we get

$$
c_{\lambda}(t)=\Gamma(t)\left(A_{\lambda}(t) c_{+_{\mathrm{D}}}+B_{\lambda}(t) c_{-t_{\mathrm{D}}}\right)+F_{\lambda}(t)
$$

where $F_{\lambda}(t)$ is the operator of the Langevin force and $\Gamma(t) \equiv \mathrm{e}^{\gamma t / 2} \mathrm{e}^{\mathrm{i}(\omega-\Delta / 2) t}$.

The time-dependent coefficients in Eq. (10) are functions of the damping rate $\gamma$ which is defined as follows,

$$
\frac{1}{2} \gamma=\pi v_{-}^{2} \sum\left|k_{j}\right|^{2} \delta\left(E_{-}-\Lambda_{j}\right)=\pi v_{+}^{2} \sum\left|k_{j}\right|^{2} \delta\left(E_{+}-\Lambda_{j}\right) .
$$

Here $\Lambda_{j}$ denotes the eigen-frequencies of the "oscillators of losses" [14] and $k_{j}$ is the coupling constant describing the mechanism of losses. As usual, we neglect the small shift of $E_{\lambda}$ [14]. There are three qualitatively different cases: (i) weak damping, when $\gamma<\Omega_{\mathrm{R}}$; (ii) critical damping, when $\gamma=\Omega_{\mathrm{R}}$; (iii) strong damping, when $\gamma>\Omega_{\mathrm{R}}$. 




Fig. 1. Dynamical behavior of the cavity photon intensity for different values of the cavity field-exciton detuning.

We have respectively

$$
\begin{aligned}
A_{+}(t) & =\cos (\xi t)-\mathrm{i}\left(\Omega_{\mathrm{R}} / 2 \xi\right) \sin (\xi t), \\
& =1-\frac{1}{2} \mathrm{i} \Omega_{\mathrm{R}} t, \\
& =\cosh (\xi t)-\mathrm{i}\left(\Omega_{\mathrm{R}} / 2 \xi\right) \sinh (\xi t), \\
B_{+}(t) & =\frac{v_{-}}{v_{+}} \frac{\gamma}{2 \xi} \sin (\xi t), \\
& =\frac{v_{-}}{v_{+}} \frac{\gamma t}{2}, \\
& =\frac{v_{-}}{v_{+}} \frac{\gamma}{2 \xi} \sinh (\xi t),
\end{aligned}
$$

and $A_{-}(t)=\left(v_{+}^{2} / v_{-}^{2}\right) B_{+}(t), B_{-}(t)=A_{+}^{*}(t)$. Here, $\xi \equiv \frac{1}{2} \sqrt{\left|\gamma^{2}-\Omega_{R}^{2}\right|}$.

We are now in a position to examine the Rabi oscillations in the system under consideration described by the periodical change of the mean number of cavity photons (7). In view of Eq. (7) we get

$$
\begin{aligned}
\left\langle c_{\lambda}^{\dagger} c_{\lambda}\right\rangle_{t}=|\Gamma(t)|^{2}\left[\left|A_{\lambda}(t)\right|^{2}\left\langle c_{+}^{\dagger} c_{+}\right\rangle_{t_{\mathrm{D}}}+\left|B_{\lambda}(t)\right|^{2}\left\langle c_{-}^{\dagger} c_{-}\right\rangle_{\mathrm{D}_{\mathrm{D}}}+2 \operatorname{Re}\left(A_{\lambda}^{*}(t) B_{\lambda}(t)\left\langle c_{+}^{\dagger} c_{-}\right\rangle_{t_{\mathrm{D}}}\right)\right], \\
2 \operatorname{Re}\left(\left\langle c_{+}^{\dagger} c_{-}\right\rangle_{r}\right)=|\Gamma(t)|^{2}\left\{2 \operatorname { R e } \left[A_{+}^{*}(t) A_{-}(t)\left\langle c_{+}^{\dagger} c_{+}\right\rangle_{t_{\mathrm{D}}}+B_{+}^{*}(t) B_{-}(t)\left\langle c_{-}^{\dagger} c_{-}\right\rangle_{t_{\mathrm{D}}}\right.\right. \\
\left.\left.+A_{+}^{*}(t) B_{-}(t)\left\langle c_{+}^{\dagger} c_{-}\right\rangle_{t_{\mathrm{D}}}+B_{+}^{*}(t) A_{-}(t)\left\langle c_{-}^{\dagger} c_{+}\right\rangle_{t_{\mathrm{D}}}\right]\right\} .
\end{aligned}
$$

According to the choice of the initial state we have

$$
\left\langle c_{\lambda}^{\dagger} c_{\lambda}\right\rangle_{t_{\mathrm{D}}}=\left|\alpha_{\lambda}\left(t_{\mathrm{D}}\right)\right|^{2}, \quad\left\langle c_{+}^{\dagger} c_{-}\right\rangle_{t_{\mathrm{D}}}=\alpha_{+}^{*}\left(t_{\mathrm{D}}\right) \alpha_{-}\left(t_{\mathrm{D}}\right) .
$$

From the definition of coefficients $A_{\lambda}(t), B_{\lambda}(t)$ it is not hard to see that the oscillating behavior of $\left\langle a^{\dagger} a\right\rangle_{\text {, }}$

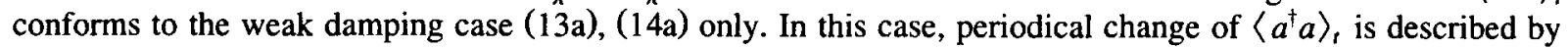




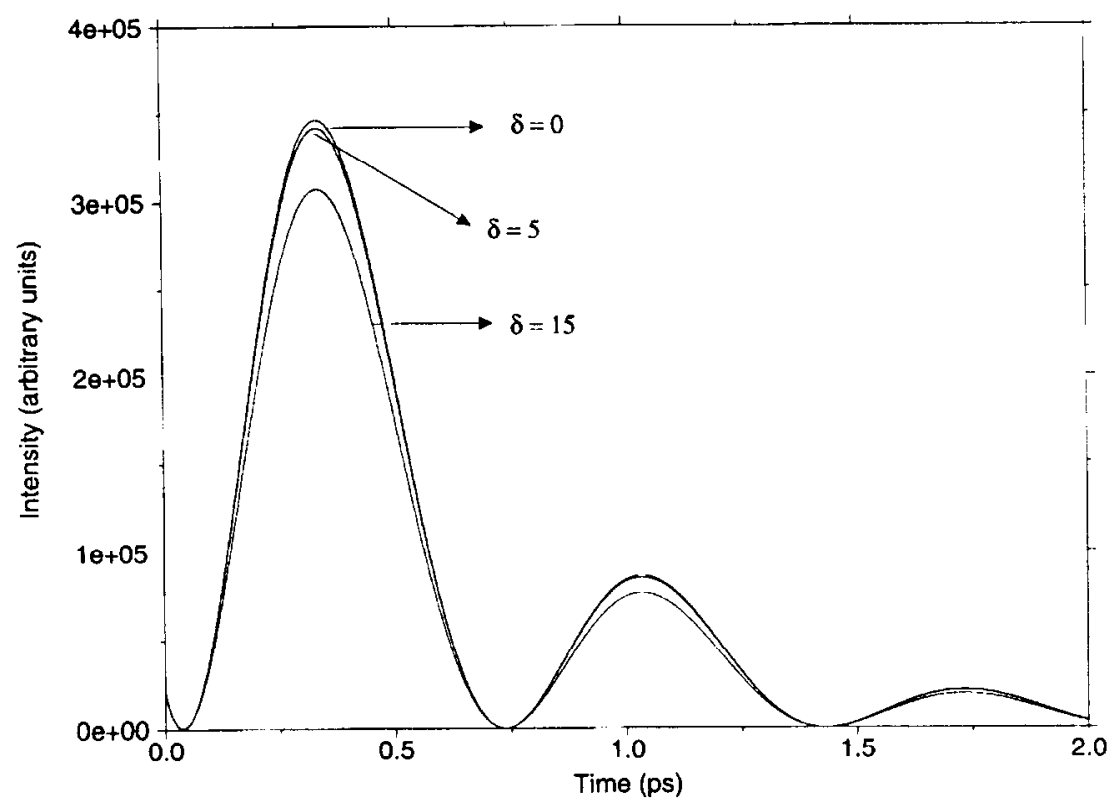

Fig. 2. Same as Fig. 1 for different values of the cavity field-pumping field detuning.

the functions $\sin ^{2}(\xi t), \cos ^{2}(\xi t), \sin (2 \xi t)$ multiplied by the corresponding coefficients and the damping factor $\mathrm{e}^{-\gamma t}$. Thus, the period of Rabi oscillations is determined by the frequency $\sqrt{\Omega_{\mathrm{R}}^{2}-\gamma^{2}}$ which plays the role of "renormalized" Rabi frequency (RRF) in the system with damping. This RRF is less than the vacuum Rabi frequency $\Omega_{\mathrm{R}}$ and tends to zero when $\gamma \rightarrow \Omega_{\mathrm{R}}$ (critical damping, (13b), (14b)). Hence, the period of Rabi oscillations in a damped cavity exceeds the period of vacuum-field-induced Rabi oscillations in an ideal cavity (3). In the case of strong damping, (13c), (14c), Rabi oscillations do not occur in the system at all.

The dependence of $\left\langle a^{\dagger} a\right\rangle$, on time in the case of weak damping, (13a), (14a), for different values of $\Delta$ is shown in Fig. 1. For numerical calculations we choose the parameters according to Ref. [9] as follows: $g=4.62 / \mathrm{ps}, t_{\mathrm{D}}=0.08 \mathrm{ps}, \epsilon=2300 / \mathrm{ps}, g \mathscr{E}_{0}=10^{4} / \mathrm{ps}, \gamma=2 / \mathrm{ps}$. In Fig. 2 we show the same as in Fig. 1 for different $\delta$ (detuning of the pumping field) at $\Delta=0$. One can see that our theoretical results show good agreement with the experimental curves [9]. Comparison of Figs. 1 and 2 shows that the detuning of the cavity mode $\Delta$ is a more critical factor for the observation of Rabi oscillations than the detuning of the pumping field $\delta$. Increase of $\Delta$ leads to a decrease of the period of the Rabi oscillations and a strong damping of the amplitude of oscillations as well.

\section{Conclusion}

Summing up our results, we should stress that the simple quantum model under consideration permits us to describe the temporal behavior of Rabi oscillations in an EP system in a quantum micro-cavity. The period of oscillations defined by the inverse value of the RRF depends on the detuning of the cavity mode and damping rate but does not depend on the detuning of the pumping field. It exceeds the period of Rabi oscillations in an ideal cavity. If the damping rate exceeds a critical value $\gamma_{c}=\sqrt{4 g^{2}+\Delta^{2}}$, the Rabi oscillations cease. At the same time, increase of $\gamma$ leads to an increase of the period of Rabi oscillations. Therefore, application of a high-quality cavity leads to an increase of the time resolution and a reduction of the duration of the pumping pulse. 
At the same time, according to condition (8) the special choice of parameters $\delta, \Delta$ and $t_{\mathrm{D}}$ can lead to a non-oscillating behavior.

The above considered model involves the most important features of the process under consideration such as photon-exciton interaction, pulse pumping, and damping. Of course, it can be supplemented by consideration of some second-order physical processes such as phonon-exciton scattering, which is important for the formation of stable states of exciton modes in a quantum well micro-cavity [13]. Inclusion of the phonon degrees of freedom can also lead to a change of the statistical properties of light emitted by the EP system $[15,16]$.

\section{Acknowledgement}

The authors would like to thank Dr. A. Silov for fruitful and stimulating discussions. A.S. would like to thank Dr. Hui Cao for a discussion of the results of Ref. [13]. Ö.M. also thanks Professor F. DeMartini for remarks.

\section{References}

[1] Y. Yamamoto, ed., Coherence, amplification, and quantum effects in semiconductor lasers (Wiley, New York, 1991).

[2] G. Rempe, H. Walther and W. Klein, Phys. Rev. Lett. 58 (1987) 353.

[3] W. Jhe, A. Anderson, E.A. Hinds, D. Meshede, L. Moi and S. Haroche, Phys. Rev. Lett. 58 (19987) 666.

[4] F. DeMartini, G. Innocenti, G.R. Jablonovitz and P. Mataloni Phys. Rev. Lett. 59 (1987) 2955.

[5] S. Mashida and Y. Yamamoto, Phys. Rev. Lett. 60 (1988) 792.

[6] H.I. Yoo and J.H. Eberly, Phys. Rep. 118 (1985) 239.

[7] H. Haken, Quantum field theory of solids (North-Holland, Amsterdam, 1983).

[8] C. Weisbuch, M. Nrefishioka, A. Ishikawa and Y. Arakawa, Phys. Rev. Lett. 69 (1992) 3314.

[9] T.B. Norris, J.K. Rhee, C.Y. Sung, Y. Arikawa, M. Nishioka and C. Weisbuch, Phys. Rev. B 50 (1994) 14663.

[10] Y. Yamamoto, F. Matinaga, S. Mashida, A. Karlsson, J. Jacobson, G. Björk and T. Mukai, J. Phys. (France) II, 3 (1993) 39.

[11] H. Cao, J. Jacobson, G. Björk, S. Pau and Y. Yamamoto, Appl. Phys. Lett. 6619951107.

[12] J. Jacobson, S. Pau, H. Cao, G. Björk and Y. Yamamoto, Phys. Rev. A 51 (1995) 2542.

[13] S. Pau, G. Björk, J. Jacobson, H. Cao and Y. Yamamoto, Phys. Rev. B 51 (1995) 7090.

[14] W.D. Louissel, Radiation and noise in quantum electronics (McGraw-Hill, New York, 1977).

[15] A.V. Chizhov, R.G. Nazmitdimov and A.S. Shumovsky, Quantum Opt. 3 (1991) 1.

[16] A.V. Chizhov, B.B. Gorkov and A.S. Shumovsky, Mod. Phys. Lett. B 7 (1993) 1233. 